\title{
Biomarkers of subclinical inflammation and increases in glycaemia, insulin resistance and beta-cell function in non-diabetic individuals: the Whitehall II study
}

\author{
Christian Herder ${ }^{1,2}$, Kristine Færch ${ }^{3}$, Maren Carstensen-Kirberg ${ }^{1,2}$, Gordon D Lowe ${ }^{4}$, \\ Rita Haapakoski ${ }^{5}$, Daniel R Witte ${ }^{6,7}$, Eric J Brunner ${ }^{5}$, Michael Roden ${ }^{1,2,8}$, \\ Adam G Tabák ${ }^{5,9}$, Mika Kivimäki ${ }^{5}$ and Dorte Vistisen ${ }^{3}$ \\ 'Institute for Clinical Diabetology, German Diabetes Center, Leibniz Center for Diabetes Research at \\ Heinrich Heine University Düsseldorf, Düsseldorf, Germany, ${ }^{2}$ German Center for Diabetes Research, \\ München-Neuherberg, Germany, ${ }^{3}$ Steno Diabetes Center, Gentofte, Denmark, ${ }^{4}$ Institute of Cardiovascular \\ and Medical Sciences, University of Glasgow, Glasgow, UK, ${ }^{5}$ Department of Epidemiology and Public Health, \\ University College London, London, UK, ${ }^{6}$ Department of Public Health, Aarhus University, Aarhus, Denmark, \\ ${ }^{7}$ Danish Diabetes Academy, Odense, Denmark, ${ }^{8}$ Department of Endocrinology and Diabetology, Medical \\ Faculty, Heinrich Heine University Düsseldorf, Düsseldorf, Germany, and ${ }^{9}$ First Department of Medicine, \\ Faculty of Medicine, Semmelweis University, Budapest, Hungary
}

Correspondence should be addressed to $\mathrm{C}$ Herder Email

christian.herder@ddz. uni-duesseldorf.de

\begin{abstract}
Objective: Higher systemic levels of pro-inflammatory biomarkers and low adiponectin are associated with increased risk of type 2 diabetes, but their associations with changes in glycaemic deterioration before onset of diabetes are poorly understood. We aimed to study whether inflammation-related biomarkers are associated with 5-year changes in glucose and insulin, $\mathrm{HbA1c}$, insulin sensitivity and beta-cell function before the diagnosis of type 2 diabetes and whether these associations may be bidirectional.

Design and methods: We used multiple repeat measures (17 891 person-examinations from 7683 non-diabetic participants) from the Whitehall II study to assess whether circulating high-sensitivity C-reactive protein (hsCRP), interleukin-6 (IL6), IL1 receptor antagonist (IL1Ra) and adiponectin are associated with subsequent changes in glycaemia, insulin, insulin resistance and beta-cell function (based on oral glucose tolerance tests). We examined bidirectionality by testing if parameters of glucose metabolism at baseline are associated with changes in inflammation-related biomarkers.

Results: Higher hsCRP and IL6 were associated with increases in fasting insulin, insulin resistance and, for IL6, with beta-cell function after adjustment for confounders. Higher adiponectin was associated with decreases in fasting glucose, HbA1c, fasting insulin, insulin resistance and beta-cell function. The reverse approach showed that 2-h glucose and insulin sensitivity were associated with changes in IL1Ra. Fasting insulin and insulin resistance showed inverse associations with changes in adiponectin.

Conclusions: Subclinical inflammation is associated with development of increased glycaemia, insulin resistance and beta-cell function in non-diabetic individuals. These findings are consistent with the hypothesis that inflammationrelated processes may increase insulin resistance and lead to a compensatory upregulation of beta-cell function.
\end{abstract}

() 2016 European Society of Endocrinology Printed in Great Britain
European Journal of

Endocrinology

(2016) 175, 367-377
Published by Bioscientifica Ltd. 


\section{Introduction}

Biomarkers of subclinical inflammation are associated with incident type 2 diabetes $(1,2)$, but prospective data on glycaemic deterioration before the onset of diabetes are scarce. Cross-sectional studies suggest differential time courses for changes in biomarkers of subclinical inflammation before type 2 diabetes. Regarding circulating C-reactive protein (CRP), for example, higher levels were observed in prediabetes (i.e., impaired fasting glucose (IFG) and/or impaired glucose tolerance (IGT)) compared with normal glucose tolerance (NGT), whereas only minor differences in CRP levels were observed between people with prediabetes and type 2 diabetes (3). In contrast, systemic levels of interleukin (IL)-6 or IL18 seemed to be similar in individuals with NGT and prediabetes, but higher in those with type 2 diabetes compared with those with prediabetes $(3,4)$. Thus, different biomarkers of subclinical inflammation are related to early vs late stages of glycaemic deterioration, but little is known about the underlying pathophysiology (5).

If subclinical inflammation influences early deterioration of glycaemic control, biomarkers of subclinical inflammation should be associated with the development of prediabetes, when individuals with NGT are followed up longitudinally. To date, two small studies have failed to provide evidence for an association of proinflammatory cytokines or adiponectin with incident IFG or IGT $(6,7)$. An alternative approach with higher statistical power is to investigate whether baseline levels of biomarkers of subclinical inflammation are associated with subsequent changes in measures of glucose metabolism $(8,9)$.

In this study, we adopted the latter approach to examine whether biomarkers of subclinical inflammation are associated with 5 -year changes in glucose and insulin levels, $\mathrm{HbA}_{1 \mathrm{c}}$, insulin sensitivity and beta-cell function before the diagnosis of type 2 diabetes in a large population-based cohort. The study was based on three 5-year observation cycles, which were combined by means of a mixed model (10). As there is evidence for an impact of hyperglycaemia and hyperinsulinaemia on subclinical inflammation and hypoadiponectinaemia $(11,12)$, we also considered a potentially bidirectional relationship by investigating to what extent markers of glucose metabolism may also be associated with changes in biomarkers of subclinical inflammation.

\section{Subjects and methods}

\section{Study participants, procedures and measurements}

Participants are from the Whitehall II study, an occupational cohort of 10308 British civil servants (6896 men and 3412 women aged $35-55$ years) of mainly white ethnicity recruited between 1985 and 1988 (phase 1) (13). The UK NHS Health Research Authority London-Harrow Ethics Committee reviewed and approved the study. Written informed consent was obtained from each participant at each examination phase. The study was conducted according to the principles of the Helsinki Declaration. The cohort has been followed at eight subsequent phases, 2.5 years apart. All study phases included a questionnaire, and every second phase (5years apart) also included a clinical health examination (phases 1 , 3, 5, 7 and 9). Phase 3 (1991-1993) was the first phase with an oral glucose tolerance test (OGTT), therefore phase 1 was not used. In the Whitehall II cohort, 8815 participated at phase 3 (1991-1993); 7870 at phase 5 (1997-1999); 6967 at phase 7 (2002-2004) and 6761 at phase 9 (2007-2009) with the same individual participating in several phases. During follow-up, participants were censored if they died, were lost to follow-up or developed diabetes. Anthropometric, demographic, clinical and lifestyle characteristics are summarised in Table 1.

At phases 3, 5, 7 and 9, a standard 2-h 75g OGTT was performed in the morning after an overnight fast $(\geq 8 \mathrm{~h}$ of fasting). For around one-third of the examinations, the OGTT was administered in the afternoon after a light fat-free breakfast ( $\geq 5 \mathrm{~h}$ of fasting). These examinations were not considered in this study. Diabetes was diagnosed by the treating physician (outside the study) or during screening by OGTT (as part of the study examination). Screen-detected diabetes was ascertained throughout follow-up by OGTTs administered every 5 years and defined according to the OGTT criteria defined by the World Health Organization (14).

Information on smoking habits (never/ex/current), alcohol consumption (units per week) and physical activity (hours per week of mild, moderate and vigorous physical activity) were collected using a self-administered questionnaire (15).

Plasma glucose, serum insulin, $\mathrm{HbA}_{1 \mathrm{c}}$ and serum lipids were measured as described previously $(16,17)$. Insulin sensitivity and beta-cell function were estimated based on fasting plasma glucose and serum insulin using the homeostasis model assessment for insulin resistance (HOMA-IR) and beta-cell function (HOMA- $\beta$ ). In addition, 
Table 1 Characteristics of the study population at each study phase. Data are presented as means (s.D.), medians (25th; 75th percentiles) or proportions $(95 \% \mathrm{Cl})$.

\begin{tabular}{l}
\hline Variable \\
\hline$n$ \\
Men (\%) \\
White ethnicity (\%) \\
Age (years) \\
BMI (kg/m²) \\
Waist circumference (cm) \\
Total cholesterol (mmol/L) \\
HDL cholesterol (mmol/L) \\
LDL cholesterol (mmol/L) \\
Triacylglycerols (mmol/L) \\
Systolic blood pressure (mmHg) \\
Diastolic blood pressure (mmHg) \\
Fasting plasma glucose (mmol/L) \\
2-h plasma glucose (mmol/L) \\
HbA ${ }_{1 c}(\%)$ \\
HbA (mmol/mol) \\
Fasting serum insulin (pmol/L) \\
2-h serum insulin (pmol/L) \\
HOMA-IR \\
HOMA- $\beta$ \\
ISI \\
hsCRP (mg/dL) \\
IL6 (pg/mL) \\
IL1Ra (ng/mL)* \\
Adiponectin ( $\mu$ g/mL)* \\
Family history of diabetes (\%) \\
Current smoker (\%) \\
Moderate-to-vigorous exercise (h/week) \\
Alcohol intake (units/week) \\
Antihypertensive treatment (\%) \\
Lipid-lowering treatment (\%) \\
\end{tabular}

Phase 3 (1991-1993)

5310
$69.1(67.8 ; 70.3)$
$90.5(89.7 ; 91.3)$
$49.4(6.0)$
$25.3(3.7)$
$85.7(11.6)$
$6.5(1.2)$
$1.4(0.4)$
$4.4(1.0)$
$1.5(1.1)$
$120.4(13.6)$
$79.7(9.5)$
$5.3(0.7)$
$5.3(1.9)$
-
-
$5.7(3.7 ; 8.9)$
$33.0(18.5 ; 56.5)$
$1.3(0.8 ; 2.1)$
$67.1(44.4 ; 100.8)$
$40.7(31.5 ; 53.3)$
$0.9(0.4 ; 1.8)$
$1.4(1.0 ; 2.0)$
$0.2(0.2 ; 0.3)$
$8.7(6.4 ; 12.4)$
$11.2(10.4 ; 12.1)$
$13.7(12.8 ; 14.7)$
$2.0(1.0 ; 5.0)$
$6.0(2.0 ; 14.0)$
$7.3(6.6 ; 8.0)$
$0.8(0.6 ; 1.1)$

\begin{tabular}{c}
\hline Phase $5(1997-1999)$ \\
\hline 4310 \\
$71.6(70.3 ; 73.0)$ \\
$91.9(91.1 ; 92.7)$ \\
$55.1(5.9)$ \\
$26.1(3.9)$ \\
$90.5(11.6)$ \\
$5.9(1.1)$ \\
$1.5(0.4)$ \\
$3.9(0.9)$ \\
$1.3(0.9)$ \\
$122.2(16.3)$ \\
$77.3(10.4)$ \\
$5.2(0.7)$ \\
$5.9(1.8)$ \\
- \\
- \\
$7.0(4.9 ; 10.2)$ \\
$32.6(19.8 ; 53.2)$ \\
$1.6(1.1 ; 2.4)$ \\
$92.0(65.1 ; 131.3)$ \\
$38.1(29.8 ; 48.9)$ \\
$1.0(0.5 ; 2.0)$ \\
$1.4(1.0 ; 2.0)$ \\
$0.3(0.3 ; 0.4)$ \\
$8.6(6.2 ; 12.2)$ \\
$10.3(9.4 ; 11.3)$ \\
$10.4(9.5 ; 11.3)$ \\
$11.5(4.5 ; 20.0)$ \\
$10.0(3.0 ; 20.0)$ \\
$11.3(10.3 ; 12.2)$ \\
$2.8(2.4 ; 3.4)$ \\
\end{tabular}

\begin{tabular}{c}
\hline Phase 7 (2002-2004) \\
\hline 4498 \\
$73.3(71.9 ; 74.5)$ \\
$92.8(92.0 ; 93.5)$ \\
$60.6(5.9)$ \\
$26.6(4.2)$ \\
$93.3(11.9)$ \\
$5.8(1.0)$ \\
$1.6(0.4)$ \\
$3.6(0.9)$ \\
$1.3(0.9)$ \\
$127.7(16.8)$ \\
$74.5(10.5)$ \\
$5.3(0.8)$ \\
$6.3(1.9)$ \\
$5.3(0.5)$ \\
$39.1(5.9)$ \\
$7.0(4.7 ; 10.7)$ \\
$37.8(23.3 ; 63.7)$ \\
$1.6(1.1 ; 2.6)$ \\
$80.0(55.2 ; 118.9)$ \\
$34.4(26.4 ; 44.5)$ \\
$1.2(0.6 ; 2.4)$ \\
$1.7(1.2 ; 2.4)$ \\
$0.3(0.3 ; 0.4)$ \\
$8.3(6.0 ; 11.7)$ \\
$10.0(9.1 ; 10.9)$ \\
$8.4(7.6 ; 9.3)$ \\
$12.0(4.5 ; 20.5)$ \\
$9.0(3.0 ; 18.0)$ \\
$22.2(21 ; 23.4)$ \\
$10.0(9.2 ; 11.0)$ \\
\hline
\end{tabular}

\begin{tabular}{c}
\hline Phase $9(2007-2009)$ \\
\hline 3773 \\
$72.9(71.4 ; 74.3)$ \\
$93.1(92.2 ; 93.8)$ \\
$65.4(5.9)$ \\
$26.6(4.3)$ \\
$94.4(11.9)$ \\
$5.3(1.1)$ \\
$1.6(0.4)$ \\
$3.2(1.0)$ \\
$1.2(0.7)$ \\
$125.3(15.9)$ \\
$71.5(10.0)$ \\
$5.2(0.6)$ \\
$6.4(1.9)$ \\
$5.6(0.4)$ \\
$43.8(5.3)$ \\
$6.6(4.3 ; 10.2)$ \\
$41.3(25.6 ; 69.1)$ \\
$1.5(1.0 ; 2.4)$ \\
$82.2(55.7 ; 120)$ \\
$33.6(25.5 ; 43.6)$ \\
- \\
- \\
$0.3(0.3 ; 0.4)$ \\
$8.5(5.6 ; 13.4)$ \\
$9.6(8.7 ; 10.6)$ \\
$5.7(5.0 ; 6.5)$ \\
- \\
$7.0(2.0 ; 16.0)$ \\
$33.0(31.5 ; 34.5)$ \\
$29.1(27.6 ; 30.6)$ \\
\hline \\
\end{tabular}

*Subsample $(n=2636)$.

whole-body insulin sensitivity was assessed using the insulin sensitivity index ( ISI $_{0,120}$ ) based on fasting and 2-h values of glucose and insulin (18).

High-sensitivity CRP (hsCRP) was measured using a high-sensitivity immunonephelometric assay, IL6 was measured using a high-sensitivity ELISA assay, IL1 receptor antagonist (IL1Ra) and total adiponectin were measured with Quantikine ELISA kits (R\&D Systems) in a diabetes case-cohort sample $(19,20)$.

\section{Statistical analysis}

Statistical analyses were performed in R, version 3.1.3 (The R Foundation for Statistical Computing) and SAS, version 9.2 (SAS Institute, Cary, NC, USA). In the main analysis, the following outcomes were studied: fasting plasma glucose, 2-h plasma glucose, $\mathrm{HbA}_{1 \mathrm{c}}$, fasting and 2-h serum insulin, HOMA-IR, HOMA- $\beta$ and $\mathrm{ISI}_{0,120}$. We excluded 10529 (36.5\%) person-examinations for which the participant had been fasting for $<8 \mathrm{~h}$ (OGTTs administered in the afternoon). Outcomes with a skewed distribution (fasting and 2-h insulin, HOMA-IR, HOMA- $\beta$ and ISI $_{0,120}$ ) were log-transformed before analysis.

The following biomarkers of subclinical inflammation were included as exposures: high-sensitivity (hs)CRP, IL6, IL1 receptor antagonist (IL1Ra) and adiponectin (all $\log 2$ transformed before analysis). As adiponectin and IL1Ra were measured only in a case-cohort subsample nested within the Whitehall II study $(19,20)$, analyses were restricted to the subcohort with these measurements. We excluded 412 (2.3\%) person-examinations with hsCRP $>10 \mathrm{mg} / \mathrm{L}$ as indicator of acute infections.

Up to a total of 17891 person-examinations for 7683 non-diabetic participants were analysed (8303 personexaminations for 2965 participants in the subcohort). We studied the associations of baseline levels of inflammationrelated biomarkers and 5-year follow-up levels of the 
Table 2 Effects (with 95\% Cl) of doubling in the inflammatory marker at baseline on 5-year changes in glycaemia, insulin, insulin sensitivity and beta-cell function.

\begin{tabular}{|c|c|c|c|c|c|c|c|}
\hline \multirow[b]{2}{*}{ Outcome } & \multirow[b]{2}{*}{ Model } & \multicolumn{3}{|c|}{ hsCRP } & \multicolumn{3}{|c|}{ IL6 } \\
\hline & & $n$ & Estimate & $P$ & $n$ & Estimate & $P$ \\
\hline \multirow[t]{4}{*}{ Fasting glucose (mmol/L) } & 1 & 6716 & $0.02(0.01 ; 0.03)$ & $<0.001$ & 6525 & $0.02(0.00 ; 0.04)$ & 0.027 \\
\hline & 2 & 6716 & $0.01(0.00 ; 0.02)$ & 0.044 & 6525 & $0.01(-0.01 ; 0.03)$ & 0.336 \\
\hline & 3 & 6716 & $0.01(0.00 ; 0.02)$ & 0.139 & 6525 & $0.00(-0.02 ; 0.02)$ & 0.778 \\
\hline & 4 & 6716 & $0.01(0.00 ; 0.02)$ & 0.208 & 6525 & $0.00(-0.02 ; 0.02)$ & 0.968 \\
\hline \multirow[t]{4}{*}{ 2-h glucose $(\mathrm{mmol} / \mathrm{L})$} & 1 & 6033 & $0.08(0.05 ; 0.10)$ & $<0.001$ & 6029 & $0.08(0.03 ; 0.13)$ & 0.003 \\
\hline & 2 & 6033 & $0.04(0.01 ; 0.07)$ & 0.004 & 6029 & $0.03(-0.02 ; 0.08)$ & 0.292 \\
\hline & 3 & 6033 & $0.03(0.00 ; 0.06)$ & 0.028 & 6029 & $0.01(-0.04 ; 0.07)$ & 0.661 \\
\hline & 4 & 6033 & $0.03(0.00 ; 0.06)$ & 0.044 & 6029 & $0.01(-0.05 ; 0.06)$ & 0.806 \\
\hline \multirow{4}{*}{$\mathrm{HbA}_{1 \mathrm{c}}(\mathrm{mmol} / \mathrm{mol})$} & 1 & 2535 & $0.06(-0.05 ; 0.16)$ & 0.285 & 2363 & $-0.11(-0.31 ; 0.08)$ & 0.263 \\
\hline & 2 & 2535 & $0.02(-0.09 ; 0.14)$ & 0.712 & 2363 & $-0.16(-0.36 ; 0.04)$ & 0.120 \\
\hline & 3 & 2535 & $0.01(-0.11 ; 0.12)$ & 0.904 & 2363 & $-0.17(-0.38 ; 0.04)$ & 0.107 \\
\hline & 4 & 2535 & $-0.01(-0.12 ; 0.11)$ & 0.874 & 2363 & $-0.22(-0.42 ;-0.02)$ & 0.033 \\
\hline \multirow[t]{4}{*}{ Fasting insulin ( $\%$ diff.) } & 1 & 6186 & $2.5(1.7 ; 3.3)$ & $<0.001$ & 6177 & $4.8(3.2 ; 6.4)$ & $<0.001$ \\
\hline & 2 & 6186 & $1.4(0.6 ; 2.3)$ & 0.001 & 6177 & $3.4(1.8 ; 5.0)$ & $<0.001$ \\
\hline & 3 & 6186 & $1.1(0.3 ; 2.0)$ & 0.010 & 6177 & $2.7(1.2 ; 4.4)$ & $<0.001$ \\
\hline & 4 & 6186 & $0.9(0.1 ; 1.7)$ & 0.024 & 6177 & $2.2(0.7 ; 3.7)$ & 0.003 \\
\hline \multirow[t]{4}{*}{ 2-h insulin (\% diff.) } & 1 & 5951 & $2.4(1.3 ; 3.5)$ & $<0.001$ & 5946 & $2.5(0.4 ; 4.8)$ & 0.021 \\
\hline & 2 & 5951 & $1.7(0.5 ; 3.0)$ & 0.005 & 5946 & $1.5(-0.7 ; 3.8)$ & 0.188 \\
\hline & 3 & 5951 & $1.4(0.2 ; 2.6)$ & 0.028 & 5946 & $0.9(-1.4 ; 3.2)$ & 0.457 \\
\hline & 4 & 5951 & $1.2(0.0 ; 2.4)$ & 0.057 & 5946 & $0.5(-1.7 ; 2.7)$ & 0.681 \\
\hline \multirow[t]{4}{*}{ HOMA-IR (\% diff.) } & 1 & 6168 & $2.7(1.8 ; 3.6)$ & $<0.001$ & 6159 & $5.0(3.3 ; 6.7)$ & $<0.001$ \\
\hline & 2 & 6168 & $1.5(0.6 ; 2.5)$ & 0.001 & 6159 & $3.5(1.8 ; 5.2)$ & $<0.001$ \\
\hline & 3 & 6168 & $1.2(0.3 ; 2.2)$ & 0.010 & 6159 & $2.7(1.0 ; 4.5)$ & 0.002 \\
\hline & 4 & 6168 & $1.0(0.1 ; 1.9)$ & 0.025 & 6159 & $2.2(0.6 ; 3.8)$ & 0.008 \\
\hline \multirow[t]{4}{*}{ HOMA- $\beta$ (\% diff.) } & 1 & 6164 & $2.1(1.3 ; 2.9)$ & $<0.001$ & 6155 & $4.3(2.7 ; 5.8)$ & $<0.001$ \\
\hline & 2 & 6164 & $1.1(0.3 ; 2.0)$ & 0.009 & 6155 & $3.0(1.5 ; 4.6)$ & $<0.001$ \\
\hline & 3 & 6164 & $1.0(0.1 ; 1.8)$ & 0.031 & 6155 & $2.6(1.0 ; 4.2)$ & 0.001 \\
\hline & 4 & 6164 & $0.8(0.0 ; 1.6)$ & 0.064 & 6155 & $2.2(0.7 ; 3.8)$ & 0.005 \\
\hline \multirow[t]{4}{*}{$\left.I S\right|_{0,120}(\%$ diff. $)$} & 1 & 5800 & $-1.6(-2.2 ;-1.0)$ & $<0.001$ & 5793 & $-1.9(-3.1 ;-0.8)$ & $<0.001$ \\
\hline & 2 & 5800 & $-1.0(-1.6 ;-0.3)$ & 0.003 & 5793 & $-1.0(-2.2 ; 0.2)$ & 0.090 \\
\hline & 3 & 5800 & $-0.8(-1.4 ;-0.1)$ & 0.022 & 5793 & $-0.6(-1.8 ; 0.6)$ & 0.320 \\
\hline & 4 & 5800 & $-0.7(-1.3 ; 0.0)$ & 0.046 & 5793 & $-0.4(-1.6 ; 0.8)$ & 0.519 \\
\hline
\end{tabular}

different outcomes, including the baseline level of the outcome as a covariate. The main analysis is based on all available data after the aforementioned exclusions and provides effect estimates per doubling in baseline levels of the respective biomarker. In addition, we used the subset of the population for whom all four biomarkers were available at the same time points to calculate regression coefficients that were standardised per 1-SD difference in the $\log$ of the biomarker to allow direct comparisons of effect sizes between the exposure variables.
All analyses were adjusted for age, sex, study phase and baseline value of the outcome studied (model 1). We further adjusted the analyses for other variables in a successive manner: model 2, further adjustment for baseline BMI; model 3, further adjustment for baseline lifestyle factors (smoking, physical activity and alcohol intake) and lipids (triacylglycerols, HDL-C and LDL-C); model 4, further adjustment for a 5-year change in BMI after baseline.

To compare the estimated associations across models 1-4 for a given outcome and exposure, we used 


\begin{tabular}{|c|c|c|c|c|c|}
\hline \multicolumn{3}{|c|}{ IL1Ra } & \multicolumn{3}{|c|}{ Adiponectin } \\
\hline$n$ & Estimate & $P$ & $n$ & Estimate & $P$ \\
\hline 3651 & $0.05(0.02 ; 0.08)$ & 0.004 & 3651 & $-0.05(-0.08 ;-0.02)$ & $<0.001$ \\
\hline 3651 & $0.03(-0.01 ; 0.06)$ & 0.132 & 3651 & $-0.04(-0.07 ;-0.01)$ & 0.003 \\
\hline 3651 & $0.02(-0.02 ; 0.05)$ & 0.353 & 3651 & $-0.04(-0.07 ;-0.01)$ & 0.020 \\
\hline 3651 & $0.01(-0.03 ; 0.05)$ & 0.591 & 3651 & $-0.04(-0.07 ;-0.01)$ & 0.011 \\
\hline 3479 & $0.11(0.01 ; 0.20)$ & 0.027 & 3479 & $-0.13(-0.21 ;-0.05)$ & 0.001 \\
\hline 3479 & $0.00(-0.10 ; 0.10)$ & 0.998 & 3479 & $-0.09(-0.17 ;-0.01)$ & 0.035 \\
\hline 3479 & $-0.03(-0.14 ; 0.07)$ & 0.509 & 3479 & $-0.07(-0.16 ; 0.02)$ & 0.135 \\
\hline 3479 & $-0.05(-0.16 ; 0.05)$ & 0.309 & 3479 & $-0.07(-0.16 ; 0.02)$ & 0.109 \\
\hline 1190 & $-0.01(-0.42 ; 0.40)$ & 0.961 & 1190 & $-0.53(-0.88 ;-0.18)$ & 0.003 \\
\hline 1190 & $-0.10(-0.55 ; 0.34)$ & 0.648 & 1190 & $-0.52(-0.88 ;-0.16)$ & 0.005 \\
\hline 1190 & $-0.23(-0.69 ; 0.23)$ & 0.325 & 1190 & $-0.45(-0.84 ;-0.06)$ & 0.026 \\
\hline 1190 & $-0.28(-0.73 ; 0.18)$ & 0.238 & 1190 & $-0.44(-0.83 ;-0.05)$ & 0.028 \\
\hline 3617 & $7.2(4.3 ; 10.2)$ & $<0.001$ & 3617 & $-5.6(-7.8 ;-3.3)$ & $<0.001$ \\
\hline 3617 & $4.9(1.9 ; 7.9)$ & 0.001 & 3617 & $-4.8(-7.0 ;-2.6)$ & $<0.001$ \\
\hline 3617 & $4.0(1.0 ; 7.1)$ & 0.009 & 3617 & $-4.1(-6.5 ;-1.7)$ & 0.001 \\
\hline 3617 & $2.4(-0.4 ; 5.3)$ & 0.094 & 3617 & $-4.6(-6.8 ;-2.3)$ & $<0.001$ \\
\hline 3428 & $4.0(0.0 ; 8.1)$ & 0.050 & 3428 & $-5.1(-8.2 ;-1.8)$ & 0.002 \\
\hline 3428 & $2.0(-2.2 ; 6.4)$ & 0.349 & 3428 & $-4.4(-7.6 ;-1.1)$ & 0.010 \\
\hline 3428 & $1.0(-3.3 ; 5.4)$ & 0.667 & 3428 & $-3.8(-7.2 ;-0.2)$ & 0.038 \\
\hline 3428 & $-0.6(-4.7 ; 3.7)$ & 0.791 & 3428 & $-4.0(-7.4 ;-0.6)$ & 0.024 \\
\hline 3612 & $7.9(4.7 ; 11.1)$ & $<0.001$ & 3612 & $-6.2(-8.6 ;-3.8)$ & $<0.001$ \\
\hline 3612 & $5.3(2.1 ; 8.7)$ & 0.001 & 3612 & $-5.4(-7.8 ;-3.0)$ & $<0.001$ \\
\hline 3612 & $4.3(1.0 ; 7.7)$ & 0.010 & 3612 & $-4.6(-7.2 ;-2.0)$ & $<0.001$ \\
\hline 3612 & $2.6(-0.5 ; 5.7)$ & 0.096 & 3612 & $-5.2(-7.5 ;-2.7)$ & $<0.001$ \\
\hline 3611 & $5.3(2.5 ; 8.2)$ & $<0.001$ & 3611 & $-3.9(-6.1 ;-1.7)$ & $<0.001$ \\
\hline 3611 & $3.7(0.8 ; 6.7)$ & 0.012 & 3611 & $-3.3(-5.5 ;-1.0)$ & 0.005 \\
\hline 3611 & $3.2(0.2 ; 6.3)$ & 0.036 & 3611 & $-2.7(-5.0 ;-0.2)$ & 0.032 \\
\hline 3611 & $2.0(-0.8 ; 5.0)$ & 0.163 & 3611 & $-3.0(-5.3 ;-0.7)$ & 0.012 \\
\hline 3419 & $-2.5(-4.5 ;-0.5)$ & 0.015 & 3419 & $2.9(1.1 ; 4.7)$ & 0.002 \\
\hline 3419 & $-0.6(-2.8 ; 1.6)$ & 0.596 & 3419 & $2.1(0.3 ; 4.0)$ & 0.023 \\
\hline 3419 & $0.0(-2.2 ; 2.4)$ & 0.970 & 3419 & $1.8(-0.2 ; 3.7)$ & 0.075 \\
\hline 3419 & $0.9(-1.4 ; 3.1)$ & 0.457 & 3419 & $1.9(0.0 ; 3.9)$ & 0.049 \\
\hline
\end{tabular}

$n$, number of person-examinations used in the particular analysis. $P, P$ value for the test of the effect being equal to zero. Values in bold indicate significant associations after adjustment for multiple testing (128 tests) with the method of Benjamini and Hochberg.

Model 1: Adjusted for baseline age, sex, study phase and baseline value of the outcome; Model 2: Further adjustment for baseline BMI; Model 3: Further adjustment for baseline smoking, physical activity, alcohol intake, triacylglycerols, HDL-C and LDL-C; Model 4: Further adjustment for 5-year change in BMI after baseline.

a complete case approach, limiting the analyses to data with complete information on all covariates in model 4. Except for $\mathrm{HbA}_{1 \mathrm{c}}$, which was only measured at phases 7 and 9, the same individual may contribute to more than one observation to the analyses. To account for the likely correlation of repeated measurements within the same participant, we used mixed-effects models with a random intercept and a random slope for time. For $\mathrm{HbA}_{1 \mathrm{c}}$, a standard linear model was used. In a sensitivity analysis, we further tested whether the associations were changed when using waist circumference instead of BMI.

In the reverse approach, we interchanged exposures and outcomes and studied the associations of the baseline levels of glycaemia, insulin, insulin sensitivity and betacell function with 5-year changes in inflammation-related biomarkers. These analyses were performed using the same methods and models as described previously.

A two-sided 5\% level of significance was adjusted for multiple testing with the method of Benjamini 
and Hochberg (21). This method controls the false discovery rate and is considered more powerful than the more simple Bonferroni adjustment of the error rate, because the risk of false negative results is lower with the Benjamini-Hochberg method.

\section{Results}

\section{Associations between biomarkers of inflammation at baseline and 5-year changes in glycaemia, insulin, insulin sensitivity and beta-cell function}

Higher systemic concentrations of hsCRP, IL6 and IL1Ra were associated with higher changes in fasting and 2-h glucose and fasting and 2-h insulin, but not $\mathrm{HbA}_{1 \mathrm{c}}$, whereas adiponectin was inversely associated with all these five outcomes (Table 2, model 1). After adjustment for baseline BMI, lipids, lifestyle factors and change in BMI, the positive associations of hsCRP and IL6 with fasting insulin and the inverse associations between adiponectin and fasting glucose, $\mathrm{HbA}_{1 \mathrm{c}}$ and fasting insulin remained significant (Table 2, models 2-4).

High baseline levels of hsCRP, IL6 and IL1Ra were also associated with increases in insulin resistance (i.e., increase in HOMA-IR and decrease in ISI $_{0,120}$ ) and betacell function, whereas baseline adiponectin showed inverse associations (Table 2, model 1). Effect sizes were attenuated by adjustment for the aforementioned covariables, but the associations of hsCRP, IL6 and adiponectin with changes in HOMA-IR and the associations of IL6 and adiponectin with HOMA- $\beta$ remained significant in the final model (model 4). Associations with ISI $_{0,120}$ lost statistical significance after adjustment.

To compare effect sizes between biomarkers of inflammation as exposure variables, we standardised our estimates per 1 population SD of 1 Log unit of the concentrations of the four biomarkers of subclinical inflammation (Fig. 1 and Supplementary Tables 1 and 2 , see section on supplementary data given at the end of this article). Effect sizes were similar for hsCRP, IL6 and IL1Ra, but of larger magnitude (and in the opposite direction) for adiponectin.

We substituted BMI with waist circumference in a sensitivity analysis. In general, this changed the effect estimates in Table 2 by $<10 \%$ (data not shown). Some effect estimates showed greater changes $(\geq 10 \%)$, but these were only observed for non-significant associations.
Associations of glycaemia, insulin, insulin sensitivity and beta-cell function at baseline with 5 -year changes in biomarkers of inflammation

When interchanging exposures and outcomes, we observed fewer significant associations (Fig. 2). None of the measures of glycaemia was associated with changes in hsCRP, IL6, IL1Ra or adiponectin when further adjusting for 5-year change in BMI after baseline (fully adjusted model), except an inverse association between 2-h glucose and IL1Ra (Supplementary Tables 3 and 4). Fasting insulin and HOMA-IR showed inverse associations with changes in adiponectin in the fully adjusted models, but neither insulin levels nor HOMA-IR were related to changes in hsCRP, IL6 or IL1Ra (Supplementary Tables 3 and 4). High baseline levels of ISI $_{0,120}$ were positively associated with increases in IL1Ra (Supplementary Tables 3 and 4).
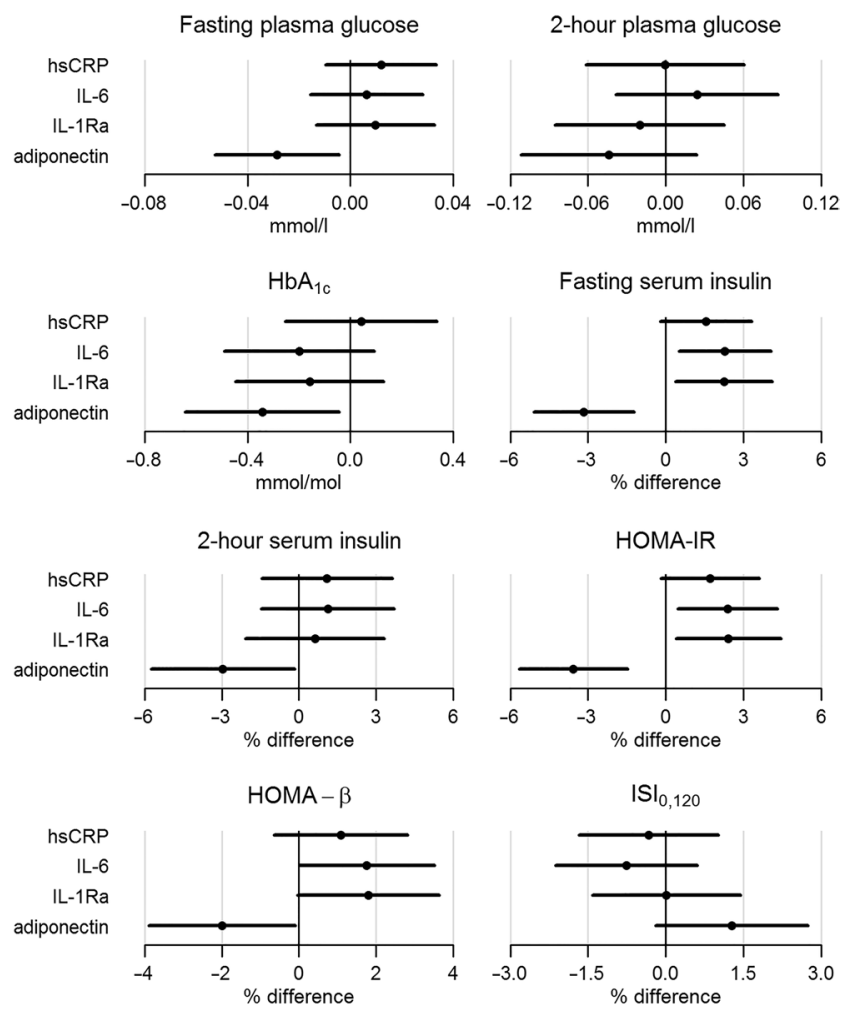

\section{Figure 1}

Effect of one population standard deviation difference in the Log of the immune marker at baseline (hsCRP, IL6, IL1RA and adiponectin) on subsequent 5-year changes in markers of glucose regulation. The associations are adjusted for baseline age, sex, study phase, BMI, smoking, physical activity, alcohol intake, triacylglycerols, HDL-C and LDL-C and baseline value of the outcome. 


\section{Discussion}

This study examined the temporal relationship between biomarkers of subclinical inflammation and changes in glucose metabolism before the diagnosis of type 2 diabetes using repeat data. Baseline levels of hsCRP and IL6 were positively associated with subsequent increases in fasting insulin, HOMA-IR and beta-cell function, whereas adiponectin was inversely associated with future changes in fasting glucose, $\mathrm{HbA}_{1 \mathrm{c}}$, fasting insulin, HOMA-IR and beta-cell function. In the reverse analysis, baseline fasting insulin and HOMA-IR were associated with decreases in adiponectin, whereas 2 -h glucose and ISI $_{0,120}$ showed associations with changes in IL1Ra.

\section{Subclinical inflammation and glycaemia}

Serum hsCRP, IL6 and IL1Ra were associated with 5-year increases in fasting and 2-h glucose in age- and sexadjusted models, but further adjustment attenuated these associations to non-significance, with BMI being the most important confounder. In contrast, adiponectin levels showed an independent inverse association with fasting glucose, but not with 2 -h glucose. These data are novel and may point towards a specific role of adiponectin in the early deterioration of glycaemia.

Fasting glucose levels are mainly determined by hepatic glucose production, whereas increased 2-h glucose
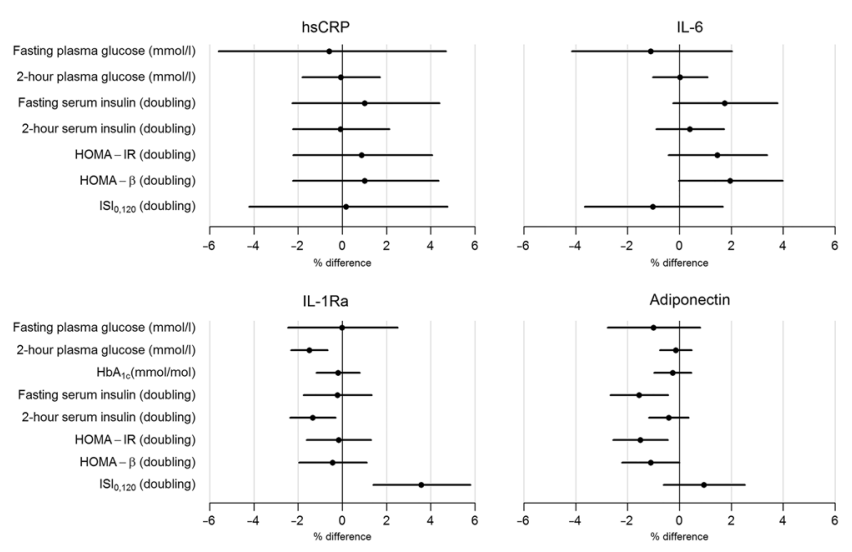

\section{Figure 2}

Effect of a difference in baseline glycaemia, insulin sensitivity or beta-cell function on subsequent 5-year changes in immune markers (hsCRP, IL6, IL1RA and adiponectin). The associations are adjusted for baseline age, sex, study phase, BMI, smoking, physical activity, alcohol intake, triacylglycerols, HDL-C and LDL-C and baseline value of the outcome. mainly reflects peripheral glucose uptake (22). Adiponectin receptors (ADIPOR)- 1 and 2 are expressed on both hepatocytes and skeletal muscle cells with ADIPOR2 being the predominant receptor in the liver and ADIPOR1 being the predominant receptor in skeletal muscle (23). Therefore, it can be speculated that ADIPOR2-mediated signalling and downstream effects on peroxisome proliferatoractivated receptor- $\alpha$ and regulation of glucose uptake, fatty acid oxidation, oxidative stress and inflammation may mediate the observed association between adiponectin and deterioration of fasting glycaemia in our study. Importantly, chronically decreased adiponectin levels are indicators of adipose tissue dysfunction and not only related to increased risk of type 2 diabetes but also related to diabetic complications $(1,2,24,25)$.

With respect to $\mathrm{HbA}_{1 \mathrm{c}}$, we observed an inverse association between adiponectin and increases in $\mathrm{HbA}_{1 \mathrm{c}}$, but no associations among the other three biomarkers. Based on the findings for fasting glucose, associations may have been expected for all four biomarkers at least for the age- and sex-adjusted model. However, this discrepancy may be because glucose levels are only weak determinants of $\mathrm{HbA}_{1 \mathrm{c}}$ in non-diabetic individuals (26). Furthermore, the sample size for the $\mathrm{HbA}_{1 \mathrm{c}}$ analysis was smaller than that for other glycaemic traits. Our data are only partly in line with previous observations in the KORA study showing a positive association between hsCRP and 7-year changes in $\mathrm{HbA}_{1 \mathrm{c}}$, but no association between adiponectin and $\mathrm{HbA}_{1 \mathrm{c}}(9)$. There are no obvious differences in baseline characteristics between the two studies, so the relevance of subclinical inflammation for $\mathrm{HbA}_{1 \mathrm{c}}$ levels in non-diabetic individuals merits further studies.

\section{Subclinical inflammation and insulin resistance}

Our study revealed consistent associations between all four biomarkers and fasting insulin and HOMA-IR, although the associations of IL1Ra were not independent of 5-year changes in BMI. In contrast, for 2-h insulin and $\mathrm{IS}_{0,120}$, which were based on post-load measures, associations with hsCRP, IL6 and adiponectin were only found in the initial regression models, but not after full adjustment.

So far, only one previous study used a comparable design and found that high hsCRP levels were associated with increases in HOMA-IR in a young non-diabetic population (8). Thus, the use of a more comprehensive assessment of subclinical inflammation and dynamic measures of insulin resistance represents an extension of the current literature. Our observations for changes in fasting insulin and HOMA-IR complemented and 
corroborated our findings for fasting glucose and pointed towards an association between subclinical inflammation and hepatic rather than peripheral insulin resistance in non-diabetic individuals. Associations were weaker for changes in IL1Ra. IL1Ra levels are considered as indicators of IL1 $\beta$-mediated processes. IL1 $\beta$ has been demonstrated to induce insulin resistance in hepatocytes (27). Therefore, an association between IL1Ra and hepatic insulin resistance is plausible.

\section{Subclinical inflammation and beta-cell function}

This is apparently the first study to show that higher hsCRP, IL6 and IL1Ra and lower adiponectin at baseline are associated with 5-year increases in beta-cell function assessed in the fasting state. After full adjustment, high IL6 levels and low adiponectin levels remained associated with increases in fasting beta-cell function.

Although an increase in beta-cell function does not seem intuitively related to an increased risk of type 2 diabetes, our findings have to be seen in context of the aforementioned associations with worsening fasting glycaemia and increased insulin resistance. The associations of IL6 and adiponectin with increases in beta-cell function were most likely a consequence of their associations with increased insulin resistance. In other words, increases in HOMA-IR in our non-diabetic study sample may reflect a compensatory upregulation of insulin secretion in response to decreases in insulin action, which was still sufficient to maintain glucose levels.

However, our data are also in line with the alternative hypothesis that biomarkers of subclinical inflammation have a direct impact on beta-cell function. IL6 has been reported to stimulate insulin secretion through an incretinmediated mechanism in experimental models of diabetes (28). The interpretation of our findings regarding beta-cell function would have been facilitated by the investigation of associations between subclinical inflammation and changes in the disposition index. Unfortunately, the assessment of dynamic beta-cell function is not possible with the available data in the Whitehall II cohort.

\section{Bidirectionality in temporal associations between subclinical inflammation and markers of glucose metabolism}

Our study is unique because our design allowed us to assess the potential bidirectionality in the associations of subclinical inflammation and glucose metabolism. Reversing our initial analysis led to two main results.
First, fasting insulin and HOMA-IR were associated with decreases in adiponectin. Secondly, 2-h glucose showed inverse and $\operatorname{ISI}_{0,120}$ showed direct associations with changes in IL1Ra.

It has been proposed that hypoadiponectinaemia in obesity and type 2 diabetes may be a consequence rather than a cause of insulin resistance (12). The regulation of adiponectin is still poorly understood in humans, so we cannot draw firm conclusions. However, the results are consistent with our previous observations of continuous and faster decrease in adiponectin levels preceding the development of type 2 diabetes compared with healthy adults (20). Our study suggests that adiponectin and insulin resistance are linked in a bidirectional way with potential deleterious consequences for the regulation of glucose metabolism.

The associations between 2-h glucose, ISI $_{0,120}$ and changes in IL1Ra point towards a potential link between peripheral insulin action and regulation of IL1Ra. Such a link is plausible as the release of both IL1 $\beta$ and IL1Ra after exercise is part of normal skeletal muscle physiology (29). However, it is unclear how impairments in muscle insulin sensitivity could influence circulating levels of both proteins.

From a pathophysiological point of view, any bidirectionality in the relationship between subclinical inflammation and insulin resistance could reflect a positive feedback loop, potentially fuelling a vicious cycle resulting in progressive worsening of glycaemic control. Our finding of a limited degree of bidirectionality consequently argues in favour of a deleterious impact of hypoadiponectinaemia and subclinical inflammation on the development of dysglycaemia.

\section{Strengths and limitations}

Strengths of our study are its large sample size and the analysis of quantitative traits entailing a larger statistical power than the analysis of a dichotomous outcome (e.g., prediabetes). Other strengths are the use of multiple measures of glucose metabolism reflecting different pathophysiological aspects and the availability of repeat data from up to four study phases, which allowed us to assess potential bidirectional relationships. Moreover, we adjusted for baseline BMI and its 5-year changes and thus demonstrated that associations were not solely mediated by obesity.

One limitation is the observational design that provides evidence for temporal, but not for causal relationships. Moreover, HOMA-IR and ISI $_{0,120}$ correlate only moderately well with the euglycaemic-hyperinsulinaemic clamp (30), 
but clamp measurements were not available. Thus, our assessment of insulin resistance was less precise than the gold standard, and we had to rely on indirect estimates to compare hepatic vs peripheral insulin resistance. HOMA- $\beta$ can only be used to estimate fasting beta-cell function, and our study did not include dynamic assessments of beta-cell function. This limits the precision of the measurement, and we could not examine beta-cell function relative to insulin sensitivity using the disposition index. We used a complete case approach in our analyses. The fraction of missingness of hsCRP and IL6 in the cohort was around $5 \%$ and between 10 and 15\% for IL1Ra and adiponectin in the subcohort. Therefore, and because we are studying associations, the effect of any potential non-randomness of the missing data for biomarkers of subclinical inflammation is considered negligible.

A final limitation of our study is the selection of four biomarkers, which left out others that also merit further research. We focused on hsCRP, IL6, IL1Ra and adiponectin as pro- and anti-inflammatory biomarkers because of their well-established associations with incident type 2 diabetes in prospective studies $(1,2,31)$. Based on experimental data and other epidemiological studies, cytokines such as $\operatorname{IL1} \beta(32,33,34)$, tumour necrosis factor (TNF)- $\alpha$ (35, 36) and transforming growth factor (TGF) $\beta(37,38)$ and chemokines such as monocyte chemoattractant protein-1/chemokine (C-C motif) ligand 2 (MCP-1/CCL2) $(39,40)$ undoubtedly represent interesting candidates because of their impact on insulin sensitivity and/or beta-cell function. However, circulating levels of IL1 $\beta$ are below the limit of detection for a large proportion of individuals in population-based studies with currently available assays, and experimental data on $\mathrm{TNF} \alpha$ and insulin resistance do not appear to be translated into an association between circulating levels of this protein and risk of type 2 diabetes in cohort studies $(41,42)$. Data on most other inflammation-related biomarkers and incident type 2 diabetes are based on only one or very few cohorts, so that further studies on their relevance both for early deterioration of glucose metabolism and for the manifestation of type 2 diabetes would be important.

\section{Conclusion}

Our study demonstrates multiple associations between baseline levels of biomarkers of subclinical inflammation and subsequent 5-year changes in glycaemia, insulin resistance and beta-cell function in a large populationbased cohort of non-diabetic individuals. These findings are consistent with the hypothesis that subclinical inflammation may increase hepatic insulin resistance and thereby upregulate beta-cell function. We observed less consistent evidence for bidirectionality in these temporal relationships, suggesting that low-grade inflammation precedes insulin resistance rather than vice versa.

\section{Supplementary data}

This is linked to the online version of the paper at http://dx.doi.org/10.1530/ EJE-16-0528.

\section{Declaration of interest}

D Vistisen and K Færch are employed by Steno Diabetes Center, a research hospital working in the Danish National Health Service and owned by Novo Nordisk A/S. Steno Diabetes Center receives part of its core funding from unrestricted grants from the Novo Nordisk Foundation and Novo Nordisk A/S. M Kivimäki reports grants from the Medical Research Council (K013351), the British Heart Foundation (RG/13/2/30098) and the US National Institutes of Health (R01 HL036310, R01AG013196), during the conduct of the study. All other authors declare that there is no duality of interest associated with their contribution to this manuscript. The funders of the study had no role in study design, data collection, analysis, interpretation or writing of the report.

\section{Funding}

This work was funded by the German Federal Ministry of Health; the Ministry of Innovation, Science, Research and Technology, North RhineWestphalia; the German Center for Diabetes Research; and the Novo Nordisk Foundation. The UK Medical Research Council, British Heart Foundation and the US National Institutes of Health (R01HL36310, R01AG013196) have supported collection of data in the Whitehall II study. D R Witte is supported by the Danish Diabetes Academy, which is funded by an unrestricted grant from the Novo Nordisk Foundation.

\section{Authors' contribution statement}

C Herder, K Færch, E J Brunner, A G Tabak, M Kivimäki and D Vistisen contributed to the study concept and design. C Herder, M CarstensenKirberg, G D Lowe, R Haapakoski, D R Witte, E J Brunner, M Roden, A G Tabak and M Kivimäki contributed the data. C Herder, K Færch and D Vistisen planned the statistical analysis. D Vistisen conducted the statistical analysis. C Herder and D Vistisen drafted the paper. All authors contributed to, critically revised and approved the final version of the manuscript.

\section{Acknowledgements}

The authors thank all the participating civil service departments and their welfare, personnel and establishment officers; the British Occupational Health and Safety Agency; the British Council of Civil Service Unions; all participating civil servants in the Whitehall II study; and all members of the Whitehall II study team. The Whitehall II study team comprises research scientists, statisticians, study coordinators, nurses, data managers, administrative assistants and data entry staff, who made the study possible.

\section{References}

1 Wang X, Bao W, Liu J, Ouyang YY, Wang D, Rong S, Xiao X, Shan ZL, Zhang Y, Yao P et al. Inflammatory markers and risk of type 2 diabetes: a systematic review and meta-analysis. Diabetes Care 2013 36 166-175. (doi:10.2337/dc12-0702) 
2 Herder C, Carstensen M \& Ouwens DM. Anti-inflammatory cytokines and risk of type 2 diabetes. Diabetes, Obesity and Metabolism 201315 (Supplement 3) 39-50. (doi:10.1111/dom.12155)

3 Grossmann V, Schmitt VH, Zeller T, Panova-Noeva M, Schulz A, Laubert-Reh D, Juenger C, Schnabel RB, Abt TG, Laskowski R et al. Profile of the immune and inflammatory response in individuals with prediabetes and type 2 diabetes. Diabetes Care 201538 1356-1364. (doi:10.2337/dc14-3008)

4 Herder C, Haastert B, Müller-Scholze S, Koenig W, Thorand B, Holle R, Wichmann HE, Scherbaum WA, Martin S \& Kolb H. Association of systemic chemokine concentrations with impaired glucose tolerance and type 2 diabetes: results from the Cooperative Health Research in the Region of Augsburg Survey S4 (KORA S4). Diabetes $2005 \mathbf{5 4}$ (Supplement 2) S11-S17. (doi:10.2337/diabetes.54.suppl_2.S11)

5 Tabák AG, Herder C, Rathmann W, Brunner EJ \& Kivimäki M. Prediabetes: a high-risk state for diabetes development. Lancet 2012 379 2279-2290. (doi:10.1016/s0140-6736(12)60283-9)

6 Daimon M, Oizumi T, Saitoh T, Kameda W, Hirata A, Yamaguchi H, Ohnuma H, Igarashi M, Tominaga M, Kato T et al. Decreased serum levels of adiponectin are a risk factor for the progression to type 2 diabetes in the Japanese population: the Funagata study. Diabetes Care 200326 2015-2020. (doi:10.2337/diacare.26.7.2015)

7 Donahue RP, Stranges S, Rejman K, Rafalson LB, Dmochowski J \& Trevisan M. Elevated cystatin C concentration and progression to pre-diabetes: the Western New York study. Diabetes Care 200730 1724-1729. (doi:10.2337/dc07-0040)

8 Park K, Steffes M, Lee DH, Himes JH \& Jacobs DR Jr. Association of inflammation with worsening HOMA-insulin resistance. Diabetologia 200952 2337-2344. (doi:10.1007/s00125-009-1486-5)

9 Klüppelholz B, Thorand B, Koenig W, de Las Heras Gala T, Meisinger C, Huth C, Giani G, Franks PW, Roden M, Rathmann W et al. Association of subclinical inflammation with deterioration of glycaemia before the diagnosis of type 2 diabetes: the KORA S4/F4 study. Diabetologia 2015 58 2269-2277. (doi:10.1007/s00125-015-3679-4)

10 Brunner EJ, Shipley MJ, Britton AR, Stansfeld SA, Heuschmann PU, Rudd AG, Wolfe CD, Singh-Manoux A \& Kivimaki M. Depressive disorder, coronary heart disease, and stroke: dose-response and reverse causation effects in the Whitehall II cohort study. European Journal of Preventive Cardiology 201421 340-346. (doi:10.1177/2047487314520785)

11 Brownlee M. The pathobiology of diabetic complications: a unifying mechanism. Diabetes 200554 1615-1625. (doi:10.2337) diabetes.54.6.1615)

12 Cook JR \& Semple RK. Hypoadiponectinemia - cause or consequence of human "insulin resistance"? Journal of Clinical Endocrinology and Metabolism 201095 1544-1554. (doi:10.1210/jc.2009-2286)

13 Marmot M \& Brunner E. Cohort profile: the Whitehall II study. International Journal of Epidemiology 200534 251-256. (doi:10.1093/ ije/dyh372)

14 Alberti KG \& Zimmet PZ. Definition, diagnosis and classification of diabetes mellitus and its complications. Part 1: diagnosis and classification of diabetes mellitus provisional report of a WHO consultation. Diabetic Medicine 199815 539-553. (doi:10.1002/ (SICI)1096-9136(199807)15:7<539::AID-DIA668>3.0.CO;2-S)

15 Stringhini S, Batty GD, Bovet P, Shipley MJ, Marmot MG, Kumari M, Tabak AG \& Kivimäki M. Association of lifecourse socioeconomic status with chronic inflammation and type 2 diabetes risk: the Whitehall II prospective cohort study. PLoS Medicine 201310 e1001479. (doi:10.1371/journal.pmed.1001479)

16 Tabák AG, Jokela M, Akbaraly TN, Brunner EJ, Kivimäki M \& Witte DR. Trajectories of glycaemia, insulin sensitivity, and insulin secretion before diagnosis of type 2 diabetes: an analysis from the Whitehall II study. Lancet 200937 2215-2221. (doi:10.1016/s01406736(09)60619-x)

17 Færch K, Witte DR, Tabák AG, Perreault L, Herder C, Brunner EJ, Kivimäki M \& Vistisen D. Trajectories of cardiometabolic risk factors before diagnosis of three subtypes of type 2 diabetes: a post-hoc analysis of the longitudinal Whitehall II cohort study. Lancet Diabetes \& Endocrinology 20131 43-51. (doi:10.1016/s2213-8587(13)70008-1)

18 Gutt M, Davis CL, Spitzer SB, Llabre MM, Kumar M, Czarnecki EM, Schneiderman N, Skyler JS \& Marks JB. Validation of the insulin sensitivity index $\left(\mathrm{ISI}_{0,120}\right)$ : comparison with other measures. Diabetes Research \& Clinical Practice 200047 177-184. (doi:10.1016/s01688227(99)00116-3)

19 Carstensen M, Herder C, Kivimäki M, Jokela M, Roden M, Shipley MJ, Witte DR, Brunner EJ \& Tabák AG. Accelerated increase in serum interleukin-1 receptor antagonist starts 6 years before diagnosis of type 2 diabetes: Whitehall II prospective cohort study. Diabetes 2010 59 1222-1227. (doi:10.2337/db09-1199)

20 Tabák AG, Carstensen M, Witte DR, Brunner EJ, Shipley MJ, Jokela M, Roden M, Kivimäki M \& Herder C. Adiponectin trajectories before type 2 diabetes diagnosis: Whitehall II study. Diabetes Care 201235 2540-2547. (doi:10.2337/dc11-2263)

21 Benjamini Y \& Hochberg Y. Controlling the false discovery rate - a practical and powerful approach to multiple testing. Journal of the Royal Statistical Society: Series B (Statistical Methodology) $1995 \mathbf{5 7}$ 289-300.

22 Abdul-Ghani MA, Tripathy D \& DeFronzo RA. Contributions of beta-cell dysfunction and insulin resistance to the pathogenesis of impaired glucose tolerance and impaired fasting glucose. Diabetes Care 200629 1130-1139. (doi:10.2337/dc05-2179)

23 Ye R \& Scherer PE. Adiponectin, driver or passenger on the road to insulin sensitivity? Molecular Metabolism 20132 133-141. (doi:10.1016/j.molmet.2013.04.001)

24 Kaptoge S, Seshasai SR, Gao P, Freitag DF, Butterworth AS, Borglykke A, Di Angelantonio E, Gudnason V, Rumley A, Lowe GD et al. Inflammatory cytokines and risk of coronary heart disease: new prospective study and updated meta-analysis. European Heart Journal 201435 578-589. (doi:10.1093/eurheartj/eht367)

25 Herder C, Bongaerts BW, Rathmann W, Heier M, Kowall B, Koenig W, Thorand B, Roden M, Meisinger C \& Ziegler D. Association of subclinical inflammation with polyneuropathy in the older population: KORA F4 study. Diabetes Care 201336 3663-3670. (doi:10.2337/dc13-0382)

26 Fizelova M, Stančáková A, Lorenzo C, Haffner SM, Cederberg H, Kuusisto J \& Laakso M. Glycated hemoglobin levels are mostly dependent on nonglycemic parameters in 9398 Finnish men without diabetes. Journal of Clinical Endocrinology and Metabolism 2015100 1989-1996. (doi:10.1210/jc.2014-4121)

27 Nov O, Kohl A, Lewis EC, Bashan N, Dvir I, Ben-Shlomo S, Fishman S, Wueest S, Konrad D \& Rudich A. Interleukin-1beta may mediate insulin resistance in liver-derived cells in response to adipocyte inflammation. Endocrinology 2010151 4247-4256. (doi:10.1210/ en.2010-0340)

28 Ellingsgaard H, Hauselmann I, Schuler B, Habib AM, Baggio LL, Meier DT, Eppler E, Bouzakri K, Wueest S, Muller YD et al. Interleukin-6 enhances insulin secretion by increasing glucagon-like peptide-1 secretion from L cells and alpha cells. Nature Medicine 2011 17 1481-1489. (doi:10.1038/nm.2513)

29 Ostrowski K, Rohde T, Asp S, Schjerling P \& Pedersen BK. Pro- and anti-inflammatory cytokine balance in strenuous exercise in humans. Journal of Physiology 1999515 287-291. (doi:10.1111/j.14697793.1999.287ad.x)

30 Simonson DC. Surrogate measures of insulin resistance: does one size fit all? Diabetologia 201558 207-210. (doi:10.1007/s00125-0143454-y)

31 Li S, Shin HJ, Ding EL \& van Dam RM. Adiponectin levels and risk of type 2 diabetes: a systematic review and meta-analysis. Journal of the American Medical Association 2009302 179-188. (doi:10.1001/ jama.2009.976)

32 Maedler K, Sergeev P, Ris F, Oberholzer J, Joller-Jemelka HI, Spinas GA, Kaiser N, Halban PA \& Donath MY. Glucose-induced beta cell 
production of IL-1beta contributes to glucotoxicity in human pancreatic islets. Journal of Clinical Investigation 2002110 851-860. (doi:10.1172/JCI200215318)

33 Jager J, Grémeaux T, Cormont M, Le Marchand-Brustel Y \& Tanti JF. Interleukin-1beta-induced insulin resistance in adipocytes through down-regulation of insulin receptor substrate-1 expression. Endocrinology 2007148 241-251. (doi:10.1210/en.2006-0692)

34 Böni-Schnetzler M \& Donath MY. Increased IL-1 $\beta$ activation, the culprit not only for defective insulin secretion but also for insulin resistance? Cell Research 201121 995-997. (doi:10.1038/cr.2011.85)

35 Hotamisligil GS, Shargill NS \& Spiegelman BM. Adipose expression of tumor necrosis factor-alpha: direct role in obesity-linked insulin resistance. Science 1993259 87-91. (doi:10.1126/science.7678183)

36 Hotamisligil GS, Murray DL, Choy LN \& Spiegelman BM. Tumor necrosis factor alpha inhibits signaling from the insulin receptor. PNAS 199491 4854-4858. (doi:10.1073/pnas.91.11.4854)

37 Herder C, Zierer A, Koenig W, Roden M, Meisinger C \& Thorand B. Transforming growth factor-beta 1 and incident type 2 diabetes: results from the MONICA/KORA case-cohort study, 1984-2002. Diabetes Care 200932 1921-1923. (doi:10.2337/dc09-0476)

38 Böhm A, Hoffmann C, Irmler M, Schneeweiss P, Schnauder G, Sailer C, Schmid V, Hudemann J, Machann J, Schick F et al. TGF $\beta$ contributes to impaired exercise response by suppression of mitochondrial key regulators in skeletal muscle. Diabetes 2016 pii: db151723. In press. (doi:10.2337/db15-1723)

39 Sell H, Dietze-Schroeder D, Kaiser U \& Eckel J. Monocyte chemotactic protein-1 is a potential player in the negative cross-talk between adipose tissue and skeletal muscle. Endocrinology 2006147 2458-2467. (doi:10.1210/en.2005-0969)

40 Herder C, Baumert J, Thorand B, Koenig W, de Jager W, Meisinger C, Illig T, Martin S \& Kolb H. Chemokines as risk factors for type 2 diabetes: results from the MONICA/KORA Augsburg study, 1984-2002. Diabetologia 200649 921-929. (doi:10.1007/s00125-006-0190-y)

41 Spranger J, Kroke A, Möhlig M, Hoffmann K, Bergmann MM, Ristow M, Boeing H \& Pfeiffer AF. Inflammatory cytokines and the risk to develop type 2 diabetes: results of the prospective populationbased European Prospective Investigation into Cancer and Nutrition (EPIC)-Potsdam Study. Diabetes 200352 812-817. (doi:10.2337/ diabetes.52.3.812)

42 Marques-Vidal P, Schmid R, Bochud M, Bastardot F, von Känel R, Paccaud F, Glaus J, Preisig M, Waeber G \& Vollenweider P. Adipocytokines, hepatic and inflammatory biomarkers and incidence of type 2 diabetes: the CoLaus study. PLoS ONE 20127 e51768. (doi:10.1371/journal.pone.0051768)

Received 21 June 2016

Revised version received 1 August 2016

Accepted 4 August 2016 